\title{
DEVELOPMENT OF THEORETICAL APPROACHES TO PHARMACEUTICAL CARE IMPROVEMENT CONSIDERING THE MODERN REQUIREMENTS OF HEALTH-CARE SYSTEM IN UKRAINE
}

\author{
IGOR A ZUPANETS ${ }^{1}$, VICTORIIA YE DOBROVA ${ }^{1}$, OLENA 0 SHILKINA ${ }^{2}$ \\ ${ }^{1}$ Department of Clinical Pharmacology and Clinical Pharmacy, National University of Pharmacy, Kharkiv, Ukraine. ${ }^{2}$ State Enterprise State \\ Expert Center of the Ministry of Health of Ukraine, Kyiv, Ukraine. Email: vd311270@gmail.com
}

Received: 19 March 2018, Revised and Accepted: 07 May 2018

ABSTRACT

Objective: The objective of this research was to formulate the theoretical approaches to the improvement of pharmaceutical care considering the modern requirements of the public health system in Ukraine.

Methods: The analysis of pharmaceutical care has been performed using "policy triangle" model. The pharmaceutical care policy model has been developed by applying the process approach.

Results: The model of pharmaceutical care as a structural element of the national health policy has been developed. This model describes mechanisms by which the content, context, and process of the pharmaceutical care policy are influenced by the content, context, and process of the national health policy. Furthermore, we have defined the actors of the pharmaceutical care policy which are groups and organizations of various levels involved in the formation and development of the pharmaceutical care policy. Then, the structure of the pharmaceutical care policy has been elaborated. This policy is integrated into the national health-care system and is adapted to the good pharmacy practice requirements. The center of the policy is a process of pharmaceutical care delivering. The inputs, outcomes, management, and resources that are required for the pharmaceutical care process and provided by the actors have been identified. The data streams within this structure demonstrate implementation of the key elements of the pharmaceutical care process: Patient involvement, patient counseling and education, interprofessional collaboration, documentation of interaction, and follow-up. Furthermore, the mechanism of continual education and increasing of the professional level has been described in this structure.

Conclusion: Proposed framework provides a comprehensive view of pharmaceutical care as a structural element of the national health policy considering new trends of the Ukrainian health system. The proposed model of the pharmaceutical care policy allows policy-makers to address all critical-to-quality aspects and stakeholders' needs.

Keywords: Pharmaceutical care, Health system, Quality improvement, Policy making.

(c) 2018 The Authors. Published by Innovare Academic Sciences Pvt Ltd. This is an open access article under the CC BY license (http://creativecommons. org/licenses/by/4. 0/) DOI: http://dx.doi.org/10.22159/ajpcr.2018.v11i8.26009

\section{INTRODUCTION}

The active process of the Ukrainian health system reformation determines the new priorities of national health-care services: Improvement of financing, quality assurance, and rational use of public costs [1]. Effectiveness, quality improvement, and patient-oriented approach have become one of the key goals of national pharmaceutical services as a result of health system reform. High-quality medication management provided by pharmacists might be considered as an element of a primary care delivery model [2]. Pharmaceutical care is an important mechanism of meeting medication-related needs of patients, particularly, within team-based collaborative drug therapy management. Thus, as a result of potential contribution to the provision of primary care, pharmaceutical care faces new challenges dictated by the changing context of the health-care system.

According to the World Health Organization (WHO)/International Pharmaceutical Federation (FIP) guidelines on good pharmacy practice (GPP), a pharmacist plays an important role in improving access to healthcare and assuring safe and efficacious use of medicines. Thus, he should be considered as a part of the comprehensive health system [3]. Joint the WHO/FIP guidelines on GPP, which outline the standards for the quality of pharmacy services, have set a new paradigm for pharmaceutical care with a patient-centered approach [3]. This concept promotes better incorporation of pharmaceutical care into public health and integration with its other elements [4].
The WHO/FIP underlines the need for collaboration of governing bodies and other health-care professional associations to provide pharmacists with continuing professional development activities and establishing national standards of pharmacy services and practice objectives. The process of setting national standards for GPP should include "a quality management framework and a strategic plan for developing services" [3]. Moreover, special attention should be paid to "both the needs of the users of health-care services and the capacity of national health-care systems to support these services" [3]. Other international guidelines also highlight the importance of patient participation and interprofessional collaboration in the improvement of pharmaceutical care [4].

To resolve these tasks, innovative system approach should be developed. This approach should provide a holistic view of pharmaceutical care within comprehensive health system considering all involved parties. Applying of this approach will allow policy-makers to design a new policy paradigm.

\section{METHODS}

Pharmaceutical care is implemented through decision-making, actions, and plans targeted on specific goals concerning health care and prevention of diseases in society. Thus, according to the WHO definition, pharmaceutical care could be considered as a health policy [5]. This allows us to analyze pharmaceutical care with appropriate research instruments. 
With the aim to determine pharmaceutical care positions within the health system, we have applied the "policy triangle" model proposed by Walt and Gilson [6]. According to this model, a health policy and its elements are analyzed by the following key parameters: (1) Actors - groups and organizations of various levels which impact the policy-making; (2) content (fundamental provisions and goals of the policy); (3) process of the actors influencing the policy formulation, implementation, and modification; and (4) context (economic, social, cultural, and other factors forming the conditions of the actors interaction and the policy-making process) $[6,7]$

To analyze pharmaceutical care and develop the model of the pharmaceutical care policy, we have applied the process approach.

\section{RESULTS}

\section{Pharmaceutical care within the national health policy}

Applying the "policy triangle" approach, we have developed the model of pharmaceutical care as a structural element of the national health policy (Fig. 1). This model demonstrably illustrates how pharmaceutical care policy is being formed under the impact of the content, context, and process of the national health policy.

Further, we will discuss in detail each of the elements of the proposed model which determine pharmaceutical care formation and development as well as its role in the national health policy.

The actors, which are in the center of the model, are groups and organizations of various levels. They are involved in the health policy making and impact the process of pharmaceutical care forming and development. Thus, the pharmaceutical care policy is being formed by state authorities, primary, the Ministry of Health of Ukraine $(\mathrm{MoH})$, as the authorized central body of the state executive authority in healthcare, and specialized governmental institutions: State enterprise State Expert Center of the $\mathrm{MoH}$, research institutions, professional organizations, biopharmaceutical companies, research and working teams, association of pharmacy workers, and patients associations. It is worth to note that activity of international organizations (for instance, the WHO), societies, and other associations also might influence the formulation of the pharmaceutical care policy at the highest level. The individuals also may act as actors involved in the process of pharmaceutical care development and implementation. In particular, they might be pharmacists, physicians, patients and pharmacy visitors, researchers, and academicians of high medical and pharmaceutical schools.

Thus, the pharmaceutical policy is being influenced at the national level in the following way: the policy content is formulated by the public health regulations, context methodology is determined by internal

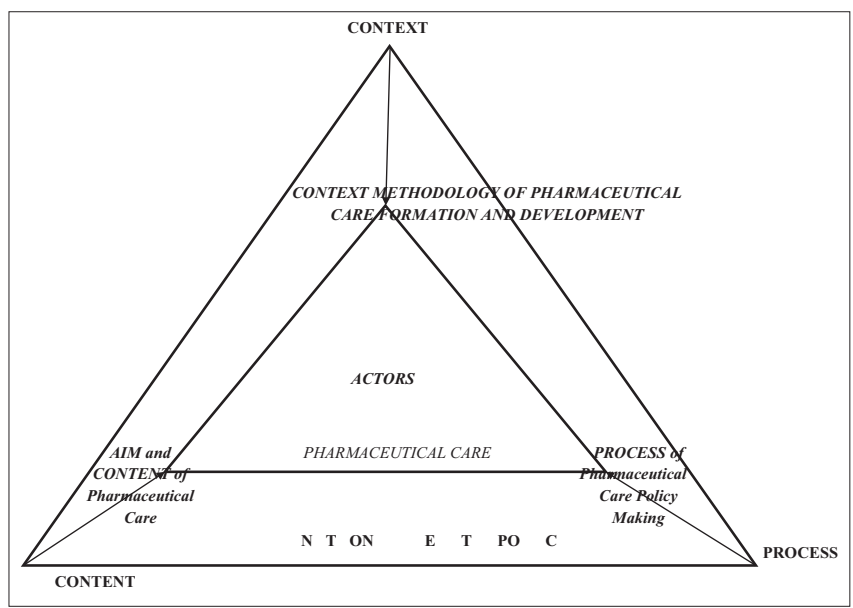

Fig. 1: The model of pharmaceutical care as a structural element of the national health policy and external factors, and the process is implemented by legislation enactment. At the same time, the policy is being impacted at the local level by medical and pharmaceutical education at high schools, postgraduate education, and training of the pharmacy workers, and, consequently, implementation of this knowledge by pharmacists interacting with the pharmacy customers. Taking this into account, it is important to analyze all elements of the pharmaceutical care policy formation both at the national and local levels of actors' impact.

The next task of this analysis is to determine the context of formation and development of the pharmaceutical care model. In this model, the context is the political, economic, and social factors that are typical for health care and pharmacy industry and creates conditions for delivering pharmaceutical care at pharmacies. Some political and economic factors influence the process of medicines licensing and, respectively, its availability at pharmacies. For instance, several years ago, all medicines containing codeine were included in the prescription medicines list. This change was caused by negative social and economic factors that, in turn, resulted in the strengthening of governmental oversight over controlled substances use and prevention of drug abuse.

Implementation of eHealth concept into pharmacy practice will improve significantly the quality of pharmaceutical services, in particular, providing pharmaceutical care. Economic factors, for example, those causing the rise of medicines prices, might hamper the accessibility of pharmaceutical care to patients and pharmacy visitors.

The health policy content determines its goals and primary tasks, as well as the procedures of pharmaceutical and health-care services delivering. This content, in turn, forms the goals and the procedures of pharmaceutical care. For instance, Ukrainian legislation establishes the licensing requirements and the procedure of pharmacies' work determines the procedures of medicines dispensing and nonprescription medicines list. Officials at the national level develop the medical and technological documentation (including pharmacist's protocols), the programs of medicines cost reimbursement, etc. The national standards regarding ethical, legal, moral, and quality aspects of health-care and pharmaceutical services delivering have influenced the formulation of ethical requirements to pharmacist's work (approval of the pharmacist ethical code) and regulate the pharmacist-physician interaction

The process of health policy making is cyclic and includes such essential phases as formulation, implementation, and modification. This process is also represented in the process of pharmaceutical care policy making. The leading trend of health system reformation is providing health-care services within the framework of evidence-based medicine. This, in turn, impacts the quality standards of pharmaceutical care, procedures of patients/customers consultation, and availability of the medicines included in the medical and technological documentation.

\section{Pharmaceutical care policy: Structure and design}

The next stage was designing the structure of pharmaceutical care policy which is integrated into the national health system and is adapted to GPP requirements (Fig. 2). To do this, we have used the list of the actors influencing pharmaceutical care policy making which were described before. All actors were classified according to the level of impact: international (regional), national, and local level.

Then, the inputs and outcomes have been identified as the basic components of this model. Inputs are required to start the pharmaceutical care process by which they are transformed into the outcomes. Outcomes are the product of the pharmaceutical care process and represent its goals. The inputs and outcomes of the pharmaceutical care process were defined according to the concept and philosophy of pharmaceutical care described by Hepler and Strand [8]. Besides, GPP requirements were also considered for inputs and outcomes identification [3] 


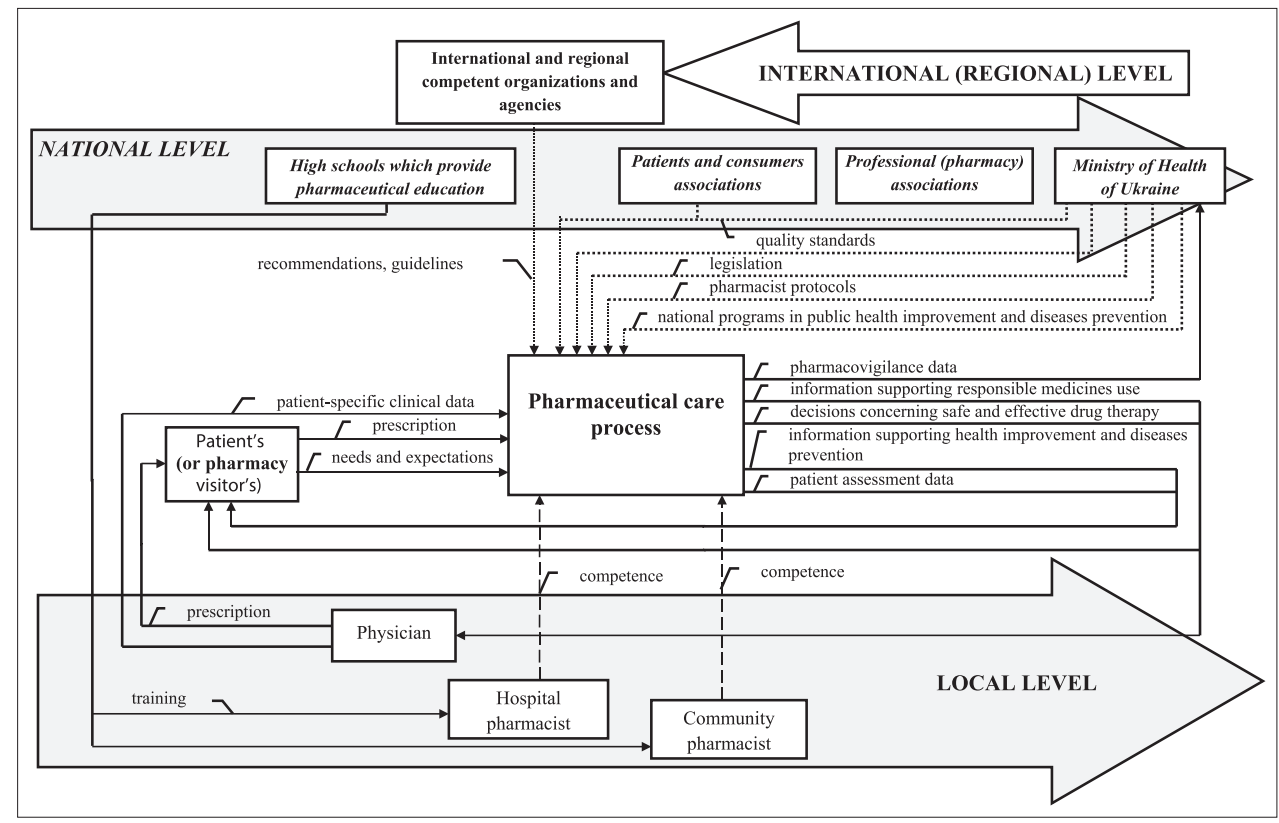

Fig. 2: The model of pharmaceutical care policy

First, patient's (or pharmacy visitor's) needs and expectations as well as physician's prescription have been defined as the inputs for the pharmaceutical care process which is transformed into the several outcomes described below.

\section{A decision concerning safe and effective medication therapy}

This outcome promotes positive results of a patient therapy. These results include curing or slowing down a disease, reduction or elimination of symptomatology, preventing a disease, or symptomatology [8]. In worth to note that important elements of this outcome are assurance cost-effectiveness of the therapy as well as preventing or/and resolving of drug-related problems [4]

\section{Information supporting responsible medicines use}

One of the key pharmacist's roles is providing consumers and prescribers with information that supports the appropriate and optimal use of medicines. In this way, a pharmacist might play a role of an educator, minimizing negative outcomes of a drug therapy such as adverse events and antimicrobial resistance.

These two outcomes, described above, go to both patients (pharmacy visitor) and a physician.

\section{Information supporting public health improvement and diseases prevention}

This is an important outcome by which a pharmacist contributes to increasing of the effectiveness of the health-care system [3,9]. This is one of the four GPP pharmacist's roles, which is a crucially important means of pharmaceutical care delivering [3]. Meeting patients' needs and expectation, pharmacist provides them with information about the safe and responsible use of medicines, self-care, and prevention care. Thus, pharmacist contributes to the national policy of promoting health in the community [3]. This outcome is consumed by a patient/ pharmacy visitor

\section{Pharmacovigilance data}

A pharmacist has a responsibility to monitor the safety of medicines use and early detection of adverse reaction. Therefore, pharmacist's contribution to drug-related problems reporting and post-approval safety monitoring is crucially important. According to FIP, "pharmacists in all practice settings are the key health professional for effective pharmacovigilance programs." Thus, this outcome is an essential product of pharmaceutical care process that is used by $\mathrm{MoH}$ for implementation of pharmacovigilance programs [10].

\section{Patient assessment data}

Tracking the patient's outcomes in the process of medication therapy, monitoring its progress, a pharmacist may contribute to assessing patient health status [3]. Besides, some diagnostic services may be available at a pharmacy (e.g., measuring blood pressure). Thus, pharmacist may control the safety of medication therapy and identify threatening states which require immediate care.

Production of these outcomes requires appropriate resources and management. At first, resources are provided by a pharmacist who has appropriate knowledge and skills. Second, a pharmacy (or a hospital where a clinical pharmacist works) provides infrastructure, equipment, and financial resources which are needed to provide patients and visitors with pharmaceutical care.

Fig. 2 shows that all inputs and resources for pharmaceutical care process are provided by the actors of local level: Pharmacists, patients, physicians, pharmacies, and hospitals. The actors of national and international (regional) levels mostly provide elements needed for pharmaceutical care management.

The MoH of Ukraine issues the orders that establish mandatory procedures for pharmaceutical care (e.g., the list of medicines approved for non-prescription dispensing), quality standards, and strategies (e.g., GPP and pharmacist's protocols) [12]. Some of these documents regulate various aspects of pharmacy practice and contain requirements to resources or/and infrastructure that support the pharmaceutical care process. Other (for instance, pharmacist's protocols) provides a detailed guideline, algorithms, and quality indicators for delivering pharmaceutical care [12]. The authority encourages the involvement of researchers, academicians, practitioners (professional associations), and representatives of the public (patients and consumers associations) in the process of these regulations development.

International organizations and agencies, such as the WHO, FIP, European Directorate for the Quality of Medicines, and others, also provide management through pharmaceutical care recommendations and guidelines. 
High schools which provide pharmaceutical education are the last group of institutions influencing pharmaceutical care policy. They provide a pharmacist with competencies during undergraduate and postgraduate education [13]. Thus, they support fulfillment of another important role of a pharmacist according to GPP requirements: Maintaining and improvement of professional performance. In terms of introduction of evidence-based medicine as a supreme basis for all therapy decisions, a pharmacist should be able to keep professional performance in accordance with new medicine achievements, to update their knowledge and be informed on new data about medical products. Therefore, the mechanism of continual education and increasing of the professional level is an important element of pharmaceutical care policy.

\section{DISCUSSION}

The proposed structure of pharmaceutical care policy allowed us to include the key components of the pharmaceutical care concept into the policy program [14]. This is necessary to assure that policy-making process will achieve all established goals of quality pharmaceutical care which would be implemented as a comprehensive, integrated system.

Patient involvement is the cornerstone of GPP that plays the crucial role in producing patient-centered pharmaceutical care policy [3,15]. Fig. 2 shows that patient's (or pharmacy visitor's) needs and expectations including their desired quality of life outcomes are the starting point of the pharmaceutical care process which initially determines its quality. It means that special attention should be paid to appropriate identification and analysis of this information. Together with appropriate evaluation of a physician's prescription (checking of accuracy, doses, incompatibility of medicines, etc.), competent evaluation of patient's (or pharmacy visitor's) needs and expectations plays a crucial role in the provision of safe and effective medication therapy [16,17]. Therefore, the agenda of the pharmaceutical care policy should include the development of the well-grounded procedure describing this process and assuring obtaining of accurate and complete information.

After competent analysis of the inputs of the pharmaceutical care process, a pharmacist is able to provide a comprehensive counseling to a patient (or pharmacy visitor) addressing identified issues $[18,19]$. Moreover, a pharmacist should inform them about responsible medicines use and self-care, preventive care activities, and services in accordance with national policies promoting the improvement of public health (role 3 in GPP guideline) [3].

Despite the widespread acknowledgment that the interprofessional collaboration is the essential condition of effective team-based care involving a pharmacist, the mechanism of pharmacist-physician interaction is vague and in fact almost inactive in Ukraine. The developed structure of the pharmaceutical care policy emphasizes the interaction between a patient, a physician, and a pharmacist (or a hospital pharmacist). Elaboration of clear and consistent procedures of this interaction is an important task of the new pharmaceutical care policy, especially considering the conditions of the health system transformation. The new health system policy includes the program of increasing primary care effectiveness and quality. Implementation of medicine cost reimbursement increases the significance of pharmacistphysician collaboration in dispensing of prescription of medicines [20].

Assessing and evaluating all papers or electronic prescriptions, a pharmacist helps to avoid medication mistakes and, in turn, will prevent the irrational use of funds allocated for reimbursement. At the same time, the probability of drug-related problem caused by inappropriate prescription will decrease, and thereby prevent additional costs of these problems treating. Thus, the procedure of pharmacist-physician collaboration in the process of prescription assessment and evaluation by a pharmacist promotes considering "the therapeutic, social, economic, and legal aspects of the prescribed indication(s)" and is an important element of quality primary care $[3,21]$. What is more, this process plays a significant role in assuring effective use of health system resources and funds allocated to the program of medicines reimbursement.

One of the new trends of the Ukrainian health system reformation is the eHealth concept. Integrating of pharmacies into this system is a crucially important condition for assuring effective physicianpharmacist collaboration and better pharmacist-patient cooperation with the purpose of achieving desired health outcomes [22]. Creating ePharmacy system within national eHealth will provide a pharmacist with tools for electronic communication with health-care providers (physician) and patients. Another important ePharmacy function is electronic dispensing that includes decisions support in handling alerts and access to an electronic patient record. Introducing of e-prescription will allow a pharmacist to receive accurate information about therapy decisions from a physician, to contribute to a development of a therapeutic plan by assessment and evaluation of a prescription, and provide more effective and quality medicines delivering to a patient.

The concept of ePharmacy system promotes implementing of GPP recommendations which stipulate that "pharmacists should have access to, contribute to, and use all necessary clinical and patient data to coordinate effective medication therapy management, especially when multiple health-care practitioners are involved in the patient's medication therapy" [3]. Access to patients records on confidentiality basis (especially on patient chronic diseases, therapy outcomes, etc.) will allow a pharmacist to provide patient-centered pharmaceutical care contributing to the advancement of pharmaceutical services, dispensing, and administrative/logistic processes [23]. Furthermore, the data generated by the eHealth system in the process of team-based therapy management and medicines dispensing will create a basis for further monitoring of the medication process.

As it was discussed before, the ePharmacy concept is an important basis ensuring feedback of pharmacist-patient (or pharmacy visitor) cooperation. After assessment of a patient's diagnosis and patientspecific needs, a pharmacist will be able to evaluate responses to administrated medications. Documenting necessary health-related data of a patient in the ePharmacy system, a pharmacist would have the ability to carry out data-based monitoring of medication therapy: to identify adverse events, track patients' therapeutic outcomes and evaluate patient progress. This mechanism will help a pharmacist to become involved in the process of disease management and provides a basis for team-based therapy management. The information obtained in the process of medication therapy monitoring is essential for assessing the need for additional medication decisions and adverse events documentation. Moreover, collecting data about adverse events and other drug-related problems by a pharmacist should be a key element of effective pharmacovigilance programs [19]. Thus, the introduction of effective information technologies (IT) into pharmacy practice will increase effectiveness and quality of pharmaceutical services. This emphasizes the conclusion that proper procedures for documentation of medication decisions and follow-up supported by appropriate IT tools should be introduced into pharmaceutical care policy.

\section{CONCLUSION}

Transformation of Ukrainian health system demands changes of current pharmacy practice approaches establishing the new trend of its development. Pharmaceutical care as a fundamental concept of pharmaceutical services should be in a line with all innovative approaches to health care such as eHealth, patient-centered approach, and effective quality management. Integrating of pharmaceutical care into the health system taking new priorities into account requires the development of the proper roadmap, application of specific approaches, as well as improvement of the pharmaceutical process itself. The latter should be substantiated by thorough analysis and rigorous framework.

Applying "policy triangle" approach enabled us to make a detailed analysis of pharmaceutical care considering fundamental aspects of the policy making, to study the specificity of pharmaceutical care policy 
formation as an element of the integrated health system, and to research its interaction within the coherent structure. Thus, pharmaceutical care has been considered comprehensively that is important for further scientifically substantiated evaluation and assurance of quality.

Analyzing pharmaceutical care as a health policy helps to elaborate a systematic approach to the understanding of its process and procedures as well as developing programs for quality improvement. In this paper, we have presented the theoretical approaches to the pharmaceutical care policy-making integrated into the health policy. These approaches provide a comprehensive view of pharmaceutical care as a structural element of national health policy.

The proposed model of the pharmaceutical care policy encompasses all elements of pharmaceutical care concept: Patient involvement, patient counseling and education, interprofessional collaboration, documentation of interaction, and follow-up. This model provides policy-makers with a framework that will help to address all criticalto-quality aspects and needs of all stakeholders. The model can be put in a basis for developing of plans and actions for pharmaceutical care quality improvement while assuring involvement of all stakeholders in the decision-making process. Considering pharmaceutical care as a process with inputs and outputs is important for the development of valid and representative quality indicators. Furthermore, this model helps to elaborate pharmaceutical care procedures in accordance with GPP guidelines which will raise the responsibility of a pharmacist and promote his involvement in disease management.

\section{AUTHOR'S CONTRIBUTIONS}

Concept and final approval of the manuscript - Igor A. Zupanets; design of the framework and critical review of the manuscript - Victoriia Ye. Dobrova; and carrying out the analysis and writing the manuscript - Olena O. Shilkina.

\section{CONFLICTS OF INTEREST}

All authors have none to declare.

\section{REFERENCES}

1. Verkhovna Rada of Ukraine. c 1994-2018. Order of the Cabinet of Ministers of Ukraine from 30.11.2016 № 1013. Available from: http:// www.zakon2.rada.gov.ua/laws/show/1013-2016-\%D1\%80. [Last cited on 2018 Mar 16].

2. ASHP. Statement on the pharmacist's role in primary care. Am J Health Syst Pharm 1999;56:1665-7.

3. International Pharmaceutical Federation. Good Pharmacy Practice Joint FIP/WHO Guidelines on GPP Standards for Quality of Pharmacy Services; c2018. Available from: http://www.fip.org/good pharmacy practice. [Last cited on 2018 Mar 16].

4. Pharmaceutical Care. Policies and Practices for a Safer, more Responsible and Cost-Effective Health System. Strasbourg: EDQM; 2012.

5. World Health Organization. Health policy; c2018. Available from: http://www.who.int/topics/health policy/en/. [Last cited on 2018 Mar 16].

6. Walt G, Gilson L. Reforming the health sector in developing countries: The central role of policy analysis. Health Policy Plan 1994;9:353-70.

7. Buse K, Nicholas M, Walt G. Making Health Policy (Understanding Public Health). 2nd ed. Maidenhead: Open University Press; 2012.

8. Hepler CD, Strand LM. Opportunities and responsibilities in pharmaceutical care. Am J Hosp Pharm 1990;47:533-43.

9. International Pharmaceutical Federation. Pharmacist ethics and Professional Autonomy: Imperatives for Keeping Pharmacy Aligned with the Public Interest; c2018. Available from: http://www.fip.org/ statements. [Last cited on 2018 Mar 16].

10. International Pharmaceutical Federation. FIP Statement of policy. The role of the pharmacist in pharmacovigilance; c2018. Available from: http://www.apps.who.int/medicinedocs/en/d/Js19754en/. [Last cited on 2018 Mar 16].

11. Blalock SJ. The effect of community pharmacy-based interventions on patient health outcomes: A systematic review. Med Care Res Rev 2016;70:235-66.

12. The State Expert Center of the Ministry of Health of Ukraine. Registry of medical and technological documentation. Pharmacist Protocol; c2018. Available from: http://www.mtd.dec.gov.ua/index.php/uk/ reiestr-mtd/item/39-protokoly-provizora-farmatsevta. [Last cited on 2018 Mar 16].

13. Palsokar G, Madhukar T. Postgraduate research in pharmaceutical sciences in India: a survey of select pharmacy professionals. Int J Curr Pharm Res 2017;9:161-4.

14. The EDQM Pharmaceutical Care Quality Indicators Project. Final Report. Strasbourg: EDQM; 2017.

15. International Pharmaceutical Federation. Statement of Professional Standards - Code of Ethics for Pharmacists; c2018. Available from: http://www.fip.org/statements. [Last cited on 2018 Mar 16].

16. Abbas A, Shah A, Khan N, Nasir H, Khan MH. Perceptions of patients' care givers regarding clinical pharmacists and their practice in a developing country. J Pharm Pharm Sci 2014;7:168-73.

17. Koehler T. Documenting the evolution of the relationship between the pharmacy support workforce and pharmacists to support patient care. Res Social Adm Pharm 2017;13:280-5.

18. Dol HS, Jadhav SS, Pisal MA, Shaikh SK, Shinde VR. Emerging trends in patient counselling: current scenario. J Pharm Pharm Sci 2015;7:658 .

19. Yang S, Kim D, Choi HJ, Chang MJ. A comparison of patients' and pharmacists' satisfaction with medication counseling provided by community pharmacies: A cross-sectional survey. BMC Health Serv Res 2016;16:131-8.

20. Schindel TJ, Yuksel N, Breault R. Perceptions of pharmacists' roles in the era of expanding scopes of practice. Res Social Adm Pharm 2017;13:148-61.

21. Famiyeh IM. Pharmacist prescribing: A scoping review about the views and experiences of patients and the public. Res Social Adm Pharm 2017;13:1-16

22. Gregorio J, Lapao LV. eHealth Pharmaceutical Services: Linking Patients, Pharmacists and Physicians - Current Strategies and Lessons Learned from ePharmacare/FCT Project Technical Report; c2018. Available from: https://www.researchgate.net/publication/304781761. [Last cited on 2018 Mar 16].

23. Pharmaceutical Care Network Europe. PCNE Workshop 4. eHealth/ ePharmacy; c2018. Available from: http://www.pcne.org/upload/ files/201_WS_4_PCNE_report.pdf. [Last cited on 2018 Mar 16]. 